\title{
QUANTUM D-MODULES, ELLIPTIC BRAID GROUPS AND DOUBLE AFFINE HECKE ALGEBRAS
}

\author{
DAVID JORDAN
}

\begin{abstract}
We build representations of the elliptic braid group from the data of a quantum $D$-module $M$ over a ribbon Hopf algebra $U$. The construction is modelled on, and generalizes, similar constructions by Lyubashenko and Majid [Ly], LyMa, and also certain geometric constructions of Calaque, Enriquez, and Etingof [CEE] concerning trigonometric Cherednik algebras. In this context, the former construction is the special case where $M$ is the basic representation, while the latter construction can be recovered as a quasi-classical limit of $U=U_{t}\left(s l_{N}\right)$, as $t \rightarrow 1$. In the latter case, we produce representations of the double affine Hecke algebra of type $A_{n-1}$, for each $n$.
\end{abstract}

\section{INTRODUCTION}

Let $G=S L_{N}(\mathbb{C})$, and $V$ be the vector representation of $G$. In [AS], Arakawa and Suzuki construct a functor $F_{n}$ from the category of $U(\mathfrak{g})$-bimodules to the category of representations of the degenerate affine Hecke algebra (AHA) $\mathcal{H}_{n}^{\text {deg }}$. Namely, as a vector space, $F_{n}(M)=\left(V^{\otimes n} \otimes M\right)^{\mathfrak{g}}$ (where $\mathfrak{g}=\operatorname{Lie}(G)$ acts on $M$ by the adjoint action), and the generators of $\mathcal{H}_{n}^{\text {deg }}$ act on $F_{n}(M)$ by certain explicit formulas.

In the paper [CEE], Calaque, Enriquez, and Etingof extended this construction to the double affine case. Namely, they upgraded the Arakawa-Suzuki functor to a functor $F_{n}$ from the category of $D_{G}$-modules to the category of representations of the degenerate (i.e., trigonometric) double affine Hecke algebra (DAHA) $\mathcal{H}_{n}^{\operatorname{deg}}(k), k=N / n$. They also considered the rational degeneration of this construction, in which one uses $D_{\mathfrak{g}}$-modules instead of $D_{G}$-modules, and the rational DAHA $\mathcal{H}_{n}^{\text {rat }}(k)$ instead of the trigonometric one.

The goal of the present paper is to generalize both of these constructions to the case of quantum groups and nondegenerate AHA and DAHA. Namely, let $U$ be a ribbon Hopf algebra with enough finite dimensional representations (i.e. the common annihilator of all finite dimensional representations is zero), and let $V$ be an irreducible finite dimensional representation of $U$. Then we define a functor $F_{n, V}$ from the category of $U$-bimodules to the category of representations of the affine braid group $B_{n}^{t r}$, given by the formula $F_{n, V}(M)=\left(V^{\otimes n} \otimes M\right)^{\text {inv }}$, where the invariants are taken with respect to the adjoint action of $U$ on $M$, and the action of the generators of the affine braid group is defined using $\mathrm{R}$ matrices. These representations are similar to those considered in EtGe]. If $V$ satisfies the Hecke condition (i.e. the braiding on $V \otimes V$ satisfies the equation $\left(\beta-q^{-1} t\right)\left(\beta+q^{-1} t^{-1}\right)=0$ ), then $F_{n, V}(M)$ descends to a representation of the AHA $\mathcal{H}_{n}(t)$. More interestingly, we upgrade the functor $F_{n, V}$ to a functor from the category of $D_{U}$-modules ( $D$-modules on 
the quantum group corresponding to $U$ ) to the category of representations of the elliptic braid group $B_{n}^{\text {ell }}$, which in the Hecke case lands in the category of representations of the DAHA $\mathcal{H}_{n}(q, t)$. If $U=U_{t}\left(s l_{N}\right), V$ the vector representation, and $t=q^{n k}$, then in the quasiclassical limit $q \rightarrow 1$ we recover the functors from [AS] and [CEE, respectively.

Our construction is also a generalization of the work of Lyubashenko and Majid [Ly], LyMa, where an action of the elliptic braid group is obtained on $\left(V^{\otimes n} \otimes A\right)^{\text {inv }}$, where $A$ is the dual Hopf algebra of $U$. Indeed, $A$ is the most basic example of a $D_{U}$-module (the module of functions on the quantum group).

Finally, we would like to discuss the connection of our paper with the work of Varagnolo and Vasserot, [VV]. This connection is the quantum counterpart of the connection between the results of [CEE] and those of [GG]. Namely, consider the setting of the present paper with $U=U_{t}\left(s l_{N}\right), V=\mathbb{C}^{N}$, and let $e_{n}$ be the Young symmetrizer of the finite Hecke algebra contained in $\mathcal{H}_{n}(q, t)$. Then the spherical subalgebra $e_{n} \mathcal{H}_{n}(q, t) e_{n}$ acts in the space $e_{n} F_{n, V}(M)=\left(S_{t}^{n} V \otimes M\right)^{\text {inv }}$ where $S_{t}^{n} V$ is the quantum symmetric power. Also, recall that Varagnolo and Vasserot define a functor $\Phi_{N, k}$ from the category of D-modules on the product $G_{t} \times \mathbb{P}_{t}^{N-1}$ of the quantum group $G_{t}$ with the quantum projective space $\mathbb{P}_{t}^{N-1}$ twisted by the $k$-th power of the line bundle $O(1)$, to the category of representations of the spherical DAHA $e_{N} \mathcal{H}_{N}\left(q, q^{k}\right) e_{N}$. It is easy to see that the space of global sections of $O(1)^{n}=O(n)$ over the quantum projective space is $S_{t}^{n} V$, so $\Phi_{N, n}(M)=e_{n} F_{n, V}(M)$, and thus through the combination of the constructions of the present paper and [VV], two different algebras $e_{n} \mathcal{H}_{n}\left(q, q^{N}\right) e_{n}$ and $e_{N} \mathcal{H}_{N}\left(q, q^{n}\right) e_{N}$ get to act on the same vector space. In a future paper, we plan to show that the images of these actions in the endomorphism algebra of this space are the same; in the rational degeneration, this was proved in [CEE]. In particular, this will imply that if $N$ divides $n$, then $e_{N} \mathcal{H}_{N}\left(q, q^{n}\right) e_{N}$ is a quotient of $e_{n} \mathcal{H}_{n}\left(q, q^{N}\right) e_{n}$. Moreover, recall from [VV2 that the algebra $e_{n} \mathcal{H}_{n}(q, t) e_{n}$ admits an "analytic continuation" with respect to $n$, which yields the quantum toroidal algebra $Q_{\lambda}(q, t)$ of type $g l(1)$, which projects to $e_{n} \mathcal{H}_{n}(q, t) e_{n}$ when $\lambda=n$ is a positive integer. The above statements should follow from the existence of an isomorphism $Q_{\lambda}\left(q, q^{\mu}\right) \rightarrow Q_{\mu}\left(q, q^{\lambda}\right)$.

In subsequent papers, we plan to study the representation-theoretic properties of the functor $F_{n, V}$, i.e. what it does to particular $U$-bimodules and $D_{U}$-modules; in particular, it would be interesting to consider the case of roots of unity. Also, our construction paves way for a number of further generalizations, which we plan to explore in the future. One of them is the quantum generalization of the paper [EFM], which generalizes the construction of CEE from the type A case to the type BC case: it defines a functor from twisted D-modules on the symmetric space $G L_{N} / G L_{p} \times G L_{q}(p+q=N)$ to representations of degenerate DAHA of type $B C_{n}$. This generalization will involve quantum symmetric spaces and the Sahi-Stokman $B C_{n}$ DAHA, and will be related to the paper [OS] in the way similar to the relation between the present paper and the construction of [VV], explained above. Another interesting direction is the generalization to the case of an arbitrary ribbon category $\mathcal{C}$, which is not necessarily the category of representations of a ribbon Hopf algebra. For example, if this category is semisimple, the role of the dual $A$ of $U$ will be played by $\oplus_{X} X \otimes X^{*}$, where $X$ runs over all simple objects of $\mathcal{C}$. In this case, if $M=A$, our 
construction would recover the natural elliptic braid group action on the genus 1 modular functor, described in the book [BK]. We note that this elliptic braid group action comes with a compatible action of the modular group $S L_{2}(\mathbb{Z})$, and we expect that under some conditions on the D-module $M$, such an action will exist on $F_{n, V}(M)$; in the Hecke case this will recover the difference Fourier transform of Cherednik Ch. Finally, we would like to use the approach of this paper to construct and study quantization of multiplicative quiver varieties of Crawley-Boevey and Shaw [CBS].

The contents of this article are laid out as follows. Section 2 consists of preliminaries. In Subsection 2.1, we recall the definitions of the elliptic braid group and a certain quotient, called the double affine Hecke Algebra. In Subsection 2.2, we recall the notion of twisting of the comultiplication of a quasi-triangular Hopf algebra $U$. This subsection is somewhat technical, and may be skipped on a first read. In Subsection 2.3, we recall the construction of the Reflection Equation algebra, which in a certain sense generalizes the algebra of functions on an algebraic group to the braided setting. To the extent possible, we give key definitions and propositions in braided-categorical terms. In Subsection 2.4, we recall the Heisenberg double construction, and its relation to differential operators on an algebraic group. In Subsection 2.5, we recall how to apply this in the non-commutative (quantum, braided) context. In particular, here is where we give the definition of quantum $D$-modules we will use in this article. In Subsection 2.6 we recall the left, right, and adjoint actions of a Lie algebra $\mathfrak{g}$ on $D_{G}$-modules, and generalize this to the quantum setting.

In Section 3, we state without proof our main results; in particular, we assert the existence of a certain family of functors from the category of quantum $D$-modules to the category of representations of the elliptic braid groups. Section 4 comprises our primary new contribution to the subject, wherein we construct the functors $F_{n, V}$ asserted in Section 3. In Section 5, we explain that the element $\tilde{Y}$ of Section 4 and a related element $\tilde{X}$ act as scalars when $V$ is irreducible. In Section 5 , we consider the case $U=U_{t}$, and we show that we can recover the geometric constructions of [CEE] as a trigonometric degeneration of our constructions.

1.1. Acknowledgments. I would like to heartily thank Pavel Etingof for explaining the construction [CEE] in the degenerate case, for his considerable help with the present construction, and for his contribution to the introduction. I would also like to thank Kobi Kremnizer for countless helpful conversations where I learned about classical and quantum $D$-modules. This project would have proceeded nowhere without their guidance. The author's work was partially supported by the NSF grant DMS-0504847.

\section{Preliminaries}

2.1. The Elliptic Braid Group and the DAHA. In this section we define the elliptic braid group following Birman and Scott $[\mathrm{Bir}]$ and $[\mathrm{Sc}]$, and a particular quotient of its group algebra, called the double affine Hecke algebra.

Definition 1. The elliptic braid group, $B_{n}^{E l l}$, is the fundamental group of the configuration space of $n$ points on the torus. It is generated by 
- the commuting elements $X_{1}, \ldots, X_{n}$

- the commuting elements $Y_{1}, \ldots, Y_{n}$

- and the braid group of the plane,

$$
B_{n}=\left\langle T_{1}, \ldots, T_{n-1} \mid \begin{array}{l}
T_{i} T_{i+1} T_{i}=T_{i+1} T_{i} T_{i+1} \forall i \\
T_{i} T_{j}=T_{j} T_{i},|i-j| \geq 2
\end{array}\right\rangle .
$$

The cross relations are:

- $T_{i} X_{i} T_{i}=X_{i+1}$,

- $T_{i} Y_{i} T_{i}=Y_{i+1}$,

- $X_{1} Y_{2}=Y_{2} X_{1} T_{1}^{2}$

- $\tilde{Y} X_{i}=X_{i} \tilde{Y}$, where $\tilde{Y}=\prod_{j} Y_{j}$.

Under the usual realization of the torus $T^{2}$ as the unit square with opposite sides glued, we choose as a basepoint the configuration with all the marked points along the diagonal. The subgroup $B_{n}$ is then identified with those braids which stay away from the sides, while the $X_{i}$ and $Y_{i}$ correspond to the horizontal and vertical global cycles, respectively. This is depicted in Figure 1, 1

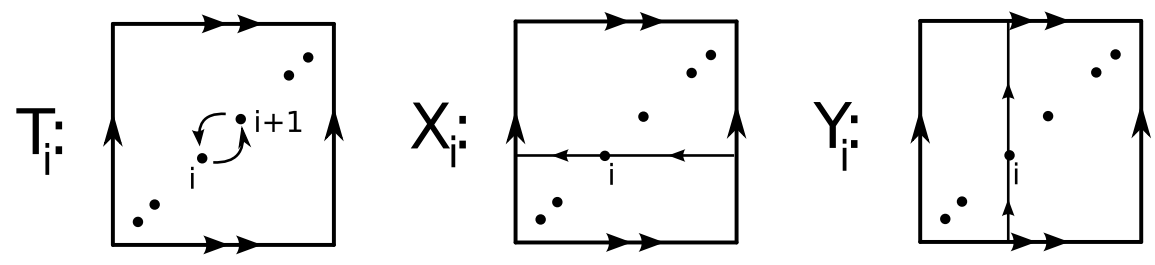

FiguRE 1. Generators for the elliptic braid group $B_{n}^{E l l}$.

Definition 2. Cherednik's double affine Hecke algebra (DAHA) $\mathcal{H}(q, t)$ is the quotient of the group algebra $\mathbb{C} B_{n}^{E l l}$ of the elliptic braid group by the additional relations

$$
\left(T_{i}-q^{-1} t\right)\left(T_{i}+q^{-1} t^{-1}\right)=0,
$$

where $q, t$ are complex parameters. Note that when $q, t=1$, we have an isomorphism $\mathcal{H H}(1,1) \cong \mathbb{C}\left[S_{n} \ltimes \mathbb{Z}^{2 n}\right]$.

Remark 1. Let us discuss the precise relation between our DAHA $\mathcal{H}_{n}(q, t)$ and the one appearing in $\mathrm{Ch}$. One can define a 3-parameter DAHA, $\mathcal{H}_{n}(q, t, p)$ by replacing the last relation of $\mathcal{H}_{n}(q, t)$ with $\tilde{Y} X_{i}=p X_{i} \tilde{Y}$. Then our algebra is $\mathcal{H}_{n}(q, t, 1)$ and Cherednik's is $\mathcal{H}_{n}(1, t, p)$.

\footnotetext{
${ }^{1}$ The reader should note that these are slightly non-standard generators (e.g. [Ch]). In particular, the $Y_{i}$ here are the inverses of the $Y_{i}$ there.
} 
QUANTUM D-MODULES, ELLIPTIC BRAID GROUPS AND DOUBLE AFFINE HECKE ALGEBRAS 5

2.2. Twistings on a Quasi-triangular Hopf algebra. Throughout this article, $U$ denotes a quasi-triangular Hopf algebra with universal $R$-matrix $\mathcal{R}=\sum_{k} r_{k}^{+} \otimes r_{k}^{-}$. We will denote by $\Delta, \epsilon, S$ the comultiplication co-unit, and antipode in $U$. Let $(\mathcal{C}, \otimes, \sigma)$ denote the braided tensor category of its left modules. Here $\sigma$ is given by $\tau \circ \mathcal{R}$, where $\tau$ is the flip of tensor factors.

We will need the Hopf algebras $U^{o}, U^{e}$, and $U^{[2]}$ as they appear in [VV]. For clarity's sake, we adopt the same notations, and recall them here.

Definition 3. Let $U^{o}$ denote the algebra $U$, with opposite co-multiplication, $\Delta^{o}(x)=$ $\tau \circ \Delta(x)$.

Definition 4. Let $U^{e}$ denote the algebra $U^{o} \otimes U$, with coordinate-wise multiplication, co-multiplication, and antipode.

Definition 5. Let $U^{[2]}$ denote the algebra $U \otimes U$ with coordinate-wise multiplication, but with comultiplication given by $\tilde{\Delta}(x \otimes y)=\mathcal{R}_{23}^{-1} \tau_{23}(\Delta(x) \otimes \Delta(y)) \mathcal{R}_{23}$, and with antipode $\tilde{S}(x \otimes y)=\mathcal{R}_{21}(S(x) \otimes S(y)) \mathcal{R}_{21}^{-1}$.

In fact, $U^{e}$ and $U^{[2]}$ are related by a 2-cocycle, which induces an equivalence on their tensor categories of modules. Let us recall these constructions:

Definition 6. Let $H$ be a Hopf algebra. A normal left 2-cocycle on $H$ is an invertible element $c \in H \otimes H$ such that

$$
(\epsilon \otimes \mathrm{id})(c)=(\mathrm{id} \otimes \epsilon)(c)=1 \otimes 1, \text { and }(\Delta \otimes \mathrm{id})(c)(c \otimes 1)=(\mathrm{id} \otimes \Delta)(c)(1 \otimes c) .
$$

A 2-cocycle is sometimes called a twist. Given a 2-cocycle $c$ on $H$, we can define the twisted Hopf algebra $H_{c}$ to be the algebra $H$ with twisted comultiplication $\Delta_{c}(h)=$ $c^{-1} \Delta(h) c$ and antipode $S_{c}(h)=Q S(h) Q^{-1}$, where $Q=\mu \circ($ id $\otimes S)(c)$. We have the following standard proposition:

Proposition 7. c induces a tensor equivalence $H$-mod $\rightarrow H_{c}$-mod.

It is now straightforward to check that $c=\mathcal{R}_{13} \mathcal{R}_{23}$ is a 2-cocycle for $U^{e}$, and that $U^{[2]}=U_{c}^{e}$.

Definition 8. Let $H$ be a Hopf algebra. An $H$-equivariant algebra $A$ is an algebra $A$ with an $H$-action on $A$ s.t. the product $\mu: A \otimes A \rightarrow A$ is a map of $H$-modules.

Remark 2. In other words, $A$ is an algebra in the category of $H$-modules.

Given an $H$-equivariant algebra $A$, we can define an $H_{c}$-equivariant algebra $A_{c}$ as the same underlying $H$-module, with multiplication given by $\mu_{c}(a \otimes b)=\mu(c(a \otimes b))$. We call $A_{c}$ the $H_{c}$-algebra equivalent to the $H$-algebra $A$.

For a $(U, U)$-bimodule, we denote the left and right actions by $\triangleright, \triangleleft($ e.g $x \triangleright m \triangleleft y)$. A $(U, U)$ bimodule is the same thing as a $U^{e}$-module under the identification $(a \otimes b)(v)=$ $b \triangleright v \triangleleft S(a)$. The co-multiplication on $U$ gives the algebra maps $\Delta: U \rightarrow U^{e}$ (resp. $U^{[2]}$ ). Thus for a $U^{e_{-}}$(resp. $\left.U^{[2]}\right]_{-}$) module $V$, we have an action of $U$, denoted "ad" given by $(\operatorname{ad} x)(v)=\Delta(x)(v)$ (we use the symbol "ad" in both contexts). 
2.3. The Reflection Equation Algebra. In this section, we will recall the so-called reflection equation algebra $A$ associated to the quasi-triangular Hopf algebra $U$. In the case that $U=U(\mathfrak{g})$ is the universal enveloping algebra of a Lie algebra $\mathfrak{g}$ of an algebraic group $G, A$ will be the algebra functions on $G$. When $U=U_{t}(\mathfrak{g})$ is the quantum group associated to the Lie algebra $\mathfrak{g}^{2}, A$ will be a braided version of the algebra of functions on $G$, distinct from the dual quantum group $O_{q}$. Majid called $A$ the "the braided Hopf algebra associated to $O_{q}$ ". The primary advantage of $A$ from our perspective is that there is an adjoint action of $U$ on $A$, for which the algebra structure on $A$ is equivariant, and which does not exist for the usual dual quantum group $O_{q}$. This equivariance property was first observed and explained by Majid [Maj], who proposed the reflection equation algebra as a preferable replacement for $O_{q}$ in the context of braided differential geometry, and showed that it was a braided-commutative braided-Hopf algebra in the category of $U$-modules.

The results here are all standard, and can be found in one form or another in many sources, e.g. Maj] or [KlSch. We include them here for completeness, and to establish the diagrammatical notation which will appear in later sections. Also, the reflection equation, Proposition 14, is usually stated for the defining modules for the FRT bialgebra, but we will need it for arbitrary modules, and so we give a diagrammatical proof.

Definition 9. Let $F$ denote the restricted dual Hopf algebra of $U$ relative to the tensor category of its finite dimensional representations. It is the sum of all finite dimensional $U^{e}$-submodules of $U^{*}$, and is spanned by functionals $c_{f, v}$, defined by $c_{f, v}(x)=f(x v)$, where $f \in V^{*}, v \in V$, and $V$ is a finite dimensional $U$-module.

One can easily check that $c_{f, v} c_{g, w}=c_{f \otimes g, v \otimes w}$, so that $F$ is a sub-algebra. The $U^{e}$-action $(x \otimes y) c_{f, v}=c_{x f, y v}$ makes $F$ into a $U^{e}$-equivariant algebra. This corresponds to the natural $(U, U)$-bimodule structure on $U^{*}$ given by $(x \triangleright \phi \triangleleft y)(h)=\phi(S(x) h y)$.

In fact, $F$ is a Hopf algebra with co-product $\Delta\left(c_{f, v}\right)=c_{f, e_{i}} \otimes c_{e_{i}^{*}, v}$, where $e_{i}$ is a basis for $V$ and $e_{i}^{*}$ is a dual basis. The antipode on $F$ is the adjoint to the antipode on $U$, defined by $\left(S c_{f, v}\right)(u)=c_{f, v}(S u)$.

Proposition 10. Let $\phi: V \rightarrow V$ be a $U$-module map, and let $\phi^{*}$ denote the adjoint map. Then $c_{f, \phi v}=c_{\phi^{*}, v}$.

Proof. For $x \in U$, we compute,

$$
c_{f, \phi v}(x)=f(x \phi(v))=f(\phi(x v))=\left(\phi^{*} f\right)(x v)=c_{\phi^{*} f, v}(x)
$$

\footnotetext{
${ }^{2}$ Note that $U_{t}$ is not quasi-triangular, since the $R$-matrix lies in a completion of $U_{t} \otimes U_{t}$. However, in all our constructions, we always apply one of the components of $\mathcal{R}$ to a finite dimensional module, so its action is well-defined.
} 
Definition 11. A dual pairing of two Hopf algebras $H$ and $K$ is a map $\kappa: H \otimes K \rightarrow \mathbb{C}$ s.t. for all $h, h^{\prime} \in H, k, k^{\prime} \in K$, we have

$$
\begin{aligned}
\kappa\left(\Delta_{H}(h), k \otimes k^{\prime}\right) & =\kappa\left(h, k k^{\prime}\right) \\
\kappa\left(h h^{\prime}, k\right) & =\kappa\left(h \otimes h^{\prime}, \Delta_{K}(k)\right) \\
\kappa\left(h, 1_{K}\right) & =\epsilon_{H}(h) \\
\kappa\left(1_{H}, k\right) & =\epsilon_{K}(k) \\
\kappa(S(h), k) & =\kappa(h, S(k))
\end{aligned}
$$

The pairing is called non-degenerate if its left and right kernels are zero.

Definition 12. We say that $U$ has enough finite-dimensional representations if the common annihilator of all finite-dimensional representations is zero.

Remark 3. The natural pairing of vector spaces $\kappa: U \otimes U^{*} \rightarrow \mathbb{C}$ restricts to a dual pairing of Hopf algebras $U$ and $F$. Since $F$ was defined as a subalgebra of $U^{*}$, the right kernel of $\kappa$ is automatically zero. The left kernel of $\kappa$ is the set of $x \in U$ s.t. $c_{f, v}(u)=0$ for all $f \in V^{*}, v \in V, V$ a f.d. $U$-module. Thus the natural pairing is non-degenerate if, and only if, $U$ has enough finite-dimensional representations. This is a mild condition which is certainly satisfied for any $U_{t}$, and which can be forced in general by quotienting $U$ by the left kernel of $\kappa$, which will be a Hopf ideal. In this case, the natural pairing between $F$ and $U$ is nondegenerate, and we get

Finite dimensional $U$-modules $\cong$ Finite dimensional $F$-comodules

We will assume from now on that $U$ has enough finite dimensional representations.

Definition 13. We denote by $A$ the $U^{[2]}$ algebra equivalent to the $U^{e}$-algebra $F$ via the cocycle $c=R_{13} R_{23}$. It has the same co-multiplication as $F$, but its multiplication $\mu^{\prime}$ is related to that of $F$ by the formula:

$$
\mu^{\prime}(f \otimes g)=\sum_{k} \mu\left(\operatorname{ad} r_{k}^{+}(f) \otimes\left(g \triangleleft S\left(r_{k}^{-}\right)\right)\right)
$$

Remark 4. For any $U$-module $V$, we have morphisms of $U$-modules

$$
c_{-,-}: V^{*} \otimes V \rightarrow A, f \otimes v \mapsto c_{f, v} .
$$

Many quantities are most easily computed in the pre-image $V^{*} \otimes V$, applying Proposition 10 as needed. In particular, the structure maps $(\mu, \Delta, S)$ for the braided-Hopf algebra $A$, as well as the proofs of Propositions 14 and 29 will be given in this way.

Remark 5. Let $V$ be a $U$-module. By duality, $V$ is an $A$-comodule, so we have a canonical element $L \in \operatorname{End}_{\mathbb{C}}(V) \otimes A$ given by $\Delta(v)=L_{1}(v) \otimes L_{2}$. This coaction may be described invariantly under the above identification as simply $\Delta: v \mapsto \sum_{i} e_{i} \otimes e_{i}^{*} v$. Here $\sum_{i} e_{i} \otimes e_{i}^{*}$ has an invariant description as the image of $1 \in \mathbb{C}$ under the coevaluation map coev $: \mathbb{C} \rightarrow$ $V \otimes V^{*}$. Similarly, formula (11) has an invariant description in terms of the braiding. These are depicted in figure 2 , 

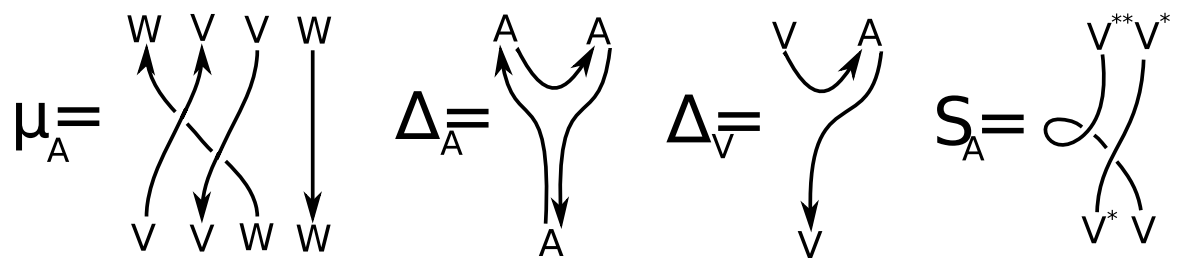

Figure 2. The multiplication and co-multiplication in $A$, the co-action on a $U$-module $V$, and the antipode in $A$. Diagrams are to be read from bottom up. The identity morphism $i d_{V}: V \rightarrow V$ is denoted with a downward flowing arrow, while $i d_{V^{*}}: V^{*} \rightarrow V^{*}$ is denoted with an upward flowing arrow. To avoid confusion, we omit arrows and explicitly label duals in the description of the antipode. An excellent reference for the diagrammatical calculus in braided tensor categories is $\mathrm{Ka}$.

Proposition 14. Maj The element $L$ defined above satisfies the reflection equation

$$
L_{01} R_{12} L_{02} R_{21}=R_{12} L_{02} R_{21} L_{01}
$$

in the space of endomorphisms of the module $V \otimes V \otimes A$. (the tensor indices run from right to left, with $A$ in index 0)

Proof. The proof is presented in figure 3 in the braided tensor category $U$-mod. The LHS diagram is $L_{01} R_{12} L_{02} R_{21}$, and the RHS is $R_{12} L_{02} R_{21} L_{01}$. Note that on the RHS we have applied Proposition 10 to the automorphism $\sigma_{V V}$ of $V \otimes V$.

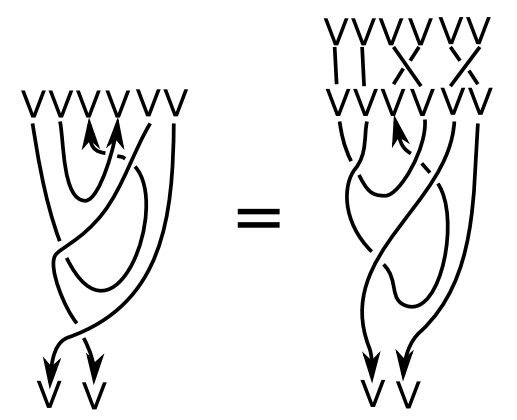

FigurE 3. A braided-categorical proof of the "Reflection Equations".

\subsection{The Heisenberg double and differential operators.}

Definition 15. Let $F$ and $U$ be dually paired Hopf algebras. Then $F$ is an $U^{o}$-module under the action $x \otimes y \mapsto \kappa\left(S(x), y_{1}\right) y_{2}$, which makes $F$ into a $U^{o}$-equivariant algebra. The Heisenberg double $H(F, U)$ is the semi-direct product of $F$ and $U^{o}$ under this action. As a vector space it is $F \otimes U$, with subalgebras $F \cong F \otimes 1, U \cong 1 \otimes U$, and cross relations

$$
x f=\left(x_{1} \triangleright f\right) x_{2}=\kappa\left(S\left(x_{1}\right), f_{1}\right) f_{2} x_{2} \text {, for all } x \in U, f \in F
$$


Example 1. D-modules on an affine algebraic group. The algebra of differential operators on an affine algebraic group has a particularly satisfactory description in terms of the Heisenberg double. Let $U=U(\mathfrak{g})$ denote the universal enveloping algebra of the Lie algebra $\mathfrak{g}$ of an algebraic group $G$, and let $O=O(G)$ denote the algebra of polynomial functions on $G$.

The Lie algebra $\mathfrak{g}$ is constructed as the sub-Lie algebra of vector fields on $G$ which are left-translation invariant. Thus we have a pairing $\kappa: U \otimes O \rightarrow \mathbb{C}$ given by $X \otimes f \mapsto X(f)_{\mathrm{id}}$. This is a dual pairing of Hopf algebras, and so we may construct the Heisenberg double $H(O, U)$. For instance, if the group $G$ is the affine plane $\mathbb{C}^{n}$, one finds $O=\mathbb{C}\left[x_{1}, \ldots, x_{n}\right]$, $U=\mathbb{C}\left[\partial_{1}, \ldots, \partial_{n}\right]$, and $H(O, U)$ is the $n$th Weyl algebra $W_{n}$. More generally, we have the following standard proposition (see, e.g. [KlSch]):

Proposition 16. The Heisenberg double $H(O, U)$ is isomorphic to the algebra $D_{G}$ of algebraic differential operators on the group $G$.

We recall the following well-known and important result:

Theorem 17. Suppose that $U$ has enough finite dimensional modules. Then $F$ is a faithful $H(F, U)$-module.

Proof. Let $\phi, \psi: F \rightarrow F$ be linear operators. Recall that the convolution $\phi * \psi: F \rightarrow F$ is defined as $(\phi * \psi)(f)=\phi\left(f_{1}\right) \psi\left(f_{2}\right)$. Then the action of $\phi:=\sum f_{i} \otimes h_{i} \in H(F, U)$ on $F$ may be written as $\rho(\phi)=\phi * 1$ (where we view $\phi$ as an element of $\operatorname{End}_{\mathbb{C}}(F)$ of finite rank, using that $\left.U \subset F^{*}\right)$ It is well known that the operator $\phi \mapsto \phi * 1$ on $\operatorname{End}_{\mathbb{C}}(F)$ is invertible for any Hopf algebra $F$, the inverse being $\phi \mapsto \phi * S$, where $S$ is the antipode. Thus $\phi * 1=0$ implies $\phi=0$, and we are done.

$F$ is sometimes called the basic representation, since when $H(F, U)$ is differential operators on an algebraic group, $F$ is just functions on the group.

2.5. Quantum $D$-modules on $U$. In this section, we want to generalize the construction of differential operators to the non-commutative setting. Our motivating example will be $U=U_{t}(\mathfrak{g})$, but we will make the construction for an arbitrary quasi-triangular Hopf algebra $U$. Key to the present construction is the isomorphism $\Xi$ constructed in [VV], which relates two potentially different notions of differential operators, one in terms of $F$, the other in terms of $A$. Indeed, it was Propositions 1.8.1 and 1.8.2 of [VV] which first alerted the author to the relevance of the reflection equation algebra $A$ to the present work.

Recall the reflection equation algebra $A$ constructed previously. As $A$ is a $U^{[2]}$-equivariant algebra, we can construct the algebra $A \rtimes U^{[2]}$.

Definition 18. $\mathrm{VV}$ The algebra $D_{U}$ of differential operators on $U$ is the subalgebra $A \otimes U \otimes 1$ of $A \rtimes U^{[2]}$. A quantum $D$-module for $U$ is a module over the algebra $D_{U}$.

Remark 6. $M$ is thus both an $A$-module and a $U$-module, such that, for $u \in U, a \in A, m \in$ $M$, we have $x(a m)=\sum_{j, k} \mu_{M}\left(\left(\left(x_{1} \otimes S\left(r_{j}^{+}\right) r_{k}^{+}\right) \triangleright a\right) \otimes r_{j}^{-} x_{2} r_{k}^{-} m\right)$. This commutation relation is depicted graphically in figure 4 . 


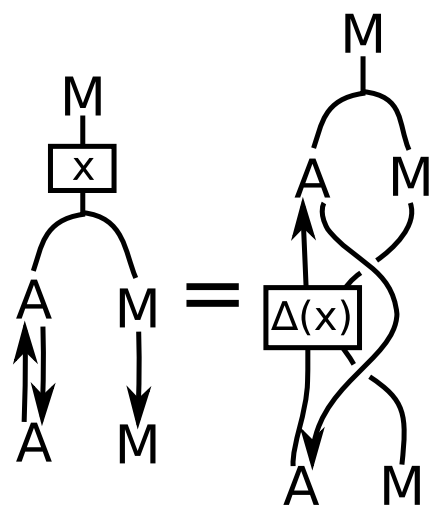

FiguRE 4. The commutation relations for differential operators.

Remark 7. Typically (e.g. $\mathrm{KrBa}$ ), a quantum $D$-module is defined as a module over the Heisenberg double $H(F, U)$. In [VV], the authors construct an isomorphism $\Xi: D_{U} \cong H(F, U)$. Thus, the present notion of a quantum $D$-module agrees with the usual notion 3

2.6. Three actions of $U$ on $D$-modules. In this section, we will recreate the left, right, and adjoint actions of vector fields on a classical $D_{G}$-module, in the non-commutative context. First, we recall the classical setup. Let $G$ be an algebraic group, and let $U=U(\mathfrak{g})$ as before. Then the left, right and adjoint action of vector fields induce algebra maps $\partial_{\triangleright}: U \rightarrow D_{U}, \partial_{\triangleleft}: U \rightarrow D_{U}$, and ad $: U \rightarrow D_{U}$. We have that $\operatorname{ad}(h)=\partial_{\triangleleft}\left(h_{1}\right) \partial_{\triangleright}\left(h_{2}\right)=$ $\mu \circ\left(\partial_{\triangleleft} \otimes \partial_{\triangleright}\right) \Delta(h)$. The assertion that ad is a homomorphism relies on the fact that the images of $\partial_{\triangleleft}$ and $\partial_{\triangleright}$ centralize one another in $D_{U}$, since the image of $\partial_{\triangleleft}$ is right invariant vector fields, and the image of $\partial_{\triangleright}$ is left invariant vector fields. A more concise way of saying this is that there is a homomorphism $\phi: U^{e} \rightarrow D_{U}$, and that the left, right, and adjoint homomorphisms are given by pre-composing with the left, right, and adjoint maps $U \rightarrow U^{e}$. Note that because we act on functions, the left action corresponds to right translation in the group, and vice versa.

In the non-commutative situation, again following [VV], we define $\partial_{\triangleleft}$ and $\partial_{\triangleright}$ as follows. We have the isomorphism $\Xi: D_{U} \cong H(F, U)$. We have the inclusion $\partial_{\triangleleft}$ as inclusion into the $U^{o}$ factor.

Definition 19. The adjoint action of $U$ on itself is given by ad : $x \otimes y \mapsto x_{1} y S\left(x_{2}\right)$.

Definition 20. Denote by $U^{\prime}$ the sub-algebra of $x \in U$ s.t. $\operatorname{ad}(U) x$ is finite dimensional.

Remark 8. It is straightforward to check that $U^{\prime}$ is indeed a subalgebra, and that $\Delta\left(U^{\prime}\right) \subset$ $U \otimes U^{\prime}$.

The adjoint action $U \otimes U^{\prime} \rightarrow U^{\prime}$ of $U$ on itself yields the co-adjoint map $\partial_{\triangleright}: U^{\prime} \rightarrow$ $F \otimes U^{\prime} \subset H(F, U)$.

\footnotetext{
${ }^{3}$ In fact, it will turn out that we could assume slightly less in our constructions: namely, we could take $M$ to be a module over the subalgebra $A \otimes U^{\prime} \subset D_{U}$ instead of the full algebra $D_{U}$, as the constructions of Section 4 only use the action of this subalgebra. However, in this article we will ignore the distinction.
} 
QUANTUM D-MODULES, ELLIPTIC BRAID GROUPS AND DOUBLE AFFINE HECKE ALGEBRAS 11

Definition 21. Let $U^{[2]^{\prime}}$ denote the subalgebra $U \otimes U^{\prime}$ of $U^{[2]}$. We define the homomorphism $\partial_{2}: U^{[2]^{\prime}} \rightarrow H\left(F, U^{o}\right)$ by $x \otimes y \mapsto \partial_{\triangleleft}(x) \partial_{\triangleright}(y)$. Abusing notation, we denote also by $\partial_{2}: U^{[2]^{\prime}} \rightarrow D_{U}$ the map $x \otimes y \mapsto \Xi^{-1}\left(\partial_{\triangleleft}(x) \partial_{\triangleright}(y)\right)$.

Remark 9. Let $z \in U$ be a central element. $z$ is thus ad-invariant, and so co-ad invariant. Thus we have that $\partial_{\triangleleft}(z)=1 \otimes z=\partial_{\triangleright}(z)$, in other words the left and right actions of $z$ agree whenever $z$ is central in $U$. We will apply this observation to the ribbon element later in the construction.

\section{Statement of Results}

Our main result is that the data of a ribbon Hopf algebra $U$, a f.d. $U$-module $V$ and a quantum $D_{U}$-module $M$ together yield representations of the elliptic braid group on $n$ strands, for any $n$. As $D_{U_{t}}$ is a flat (in fact, trivial) deformation of $D_{G}$, this provides a rich source of such representations. Taking $M$ to be the "basic" $D_{U}$-module $A$, we recover the construction of Lyubashenko and Majid [Ly, LyMa. Alternatively, taking a quasi-classical limit as $t \rightarrow 1$ for the quantum group $U_{t}\left(s l_{N}\right)$, we recover the geometric constructions from [CEE]. Thus our results are really an interpolation of those two papers. In a future paper, we hope to elaborate the case $U=U_{t}\left(s l_{N}\right)$ in greater detail, and extend some representation-theoretic results of [CEE].

To state the main theorem, we need to introduce some further notation. Let $Z$ be a $U$-module. We denote by $Z^{i n v}$ the vector space

$$
Z^{i n v}=\operatorname{Hom}_{U}(1, Z)=\{w \in Z \text { s.t. } x w=\epsilon(x) w \forall x \in U\} .
$$

Our main result is the following:

Theorem 22. Let $U$ be a ribbon Hopf algebra. Let $n \in \mathbb{N}, V$ a f.d. U-module, and $M a$ $D_{U}$-module. On the vector space

$$
W=\left(V^{\otimes n} \otimes M\right)^{i n v}
$$

of invariants w.r.t to the adjoint action on $M$, we have an action of the elliptic braid group $B_{n}^{\text {Ell }}$, which defines a functor $F_{n, V}: D_{U}-\bmod \rightarrow \operatorname{Rep}\left(B_{n}^{E l l}\right)$.

We will provide the construction in Section 4, from which will follow two easy corollaries:

Corollary 23. Let $\nu \in U$ be the ribbon element. Suppose that $V$ is irreducible, and that $\left.\nu\right|_{V}=c_{V} i d_{V}$. Then, $\left.\tilde{Y}\right|_{V}=\left.\tilde{X}\right|_{V}=c_{V}^{n} i d_{W}$.

Corollary 24. Suppose the braiding on $V$ satisfies the Hecke relation

$$
\left(\sigma_{V V}-q^{-1} t\right)\left(\sigma_{V V}+q^{-1} t^{-1}\right) .
$$

Then the action of $B_{n}^{E l l}$ descends to an action of the double affine Hecke algebra (DAHA) $\mathcal{H}_{n}(q, t)$, and we have a functor $F_{n, V}: D_{U}-\bmod \rightarrow \operatorname{Rep}\left(\mathcal{H}_{n}(q, t)\right)$.

Finally, we consider the example $U=U_{t}\left(s l_{N}\right)$, and $V$ is the defining representation, of highest weight $(1,0, \ldots, 0)$. It is well-known that $V$ is Hecke with parameters $q, t=$ $q^{n k}, k=N / n$. In [CEE], the authors considered $D$-modules on (the classical group) $S L_{N}$; 
given a $D$-module $M$, they constructed a representation of the trigonometric Cherednik algebra $\mathcal{H}_{n}^{\text {deg }}(k)$ of type $A_{n-1}$ on the space $\left(V^{\otimes n} \otimes M\right)^{i n v}$, with parameter $k=N / n$. As $\mathcal{H}_{n}^{\text {deg }}(k)$ is the quasi-classical limit of the DAHA as the parameter $t \rightarrow 1$, we can ask whether our construction agrees with theirs in the quasi-classical limit. Indeed, we have the following

Theorem 25. In the quasi-classical limit as $t \rightarrow 1$ the construction in Proposition 24 recovers the $\mathcal{H}_{n}^{\text {deg }}(k)$-representations constructed in [CEE].

If we forget the $A$-action, and consider $M$ only as a $U^{e}$-module, i.e. a $U$-bimodule, we can still define the operators $Y_{i}, T_{j}$. In fact, we have the following result which follows from the proof of Theorem 22

Corollary 26. Let $M$ be a $U^{e}$-module. The operators $Y_{i}, T_{j}$ define an action of the affine Hecke algebra on $W$, whose quasi-classical limit is the Arakawa-Suzuki construction from AS.

\section{The Construction}

This entire section is devoted to a constructive proof of Theorem 22, We will build the representation of $B_{n}^{E l l}$ by constructing first the braid group representation, then the action of the algebra $\mathbb{C}[Y]$, then the algebra $\mathbb{C}[X]$, and finally checking the commutation relations between them.

Fix $U$, a ribbon Hopf algebra with ribbon element $\nu$, and let $A$ and $D_{U}$ be as in Definitions 13 and 18, Let $M$ be a $D_{U}$-module, let $V$ be a $U$-module, and consider the vector space

$$
W=\left(V^{\otimes n} \otimes M\right)^{i n v} .
$$

This means that we take invariants with respect to the usual action on the $V^{\otimes n}$ factor, and with respect to the adjoint action on the $M$ factor. It is important to note here that the adjoint action is only defined for elements of $U^{\prime}$, the locally finite part of $U$. So we will only be able to apply ad-invariance for such elements.

We index the tensor factors in $W$ from right to left, starting with $M$ at index 0 . We will use the symbol $\pi_{V}$ when we want to explicitly emphasize the action on $V$.

The action of the braid group is given by $T_{i}=\sigma_{i+1, i}$ acting on the $V^{\otimes n}$ factor. We define the invertible element $Y_{1}=\sigma_{M, V} \circ \sigma_{V, M}=\left(\pi_{V} \otimes \partial_{\triangleleft}\right)\left(R_{01} R_{10}\right)$ acting on the right two factors. We define operators $Y_{i}$, for $i=1, \ldots, n-1$ by $Y_{i+1}=T_{i} Y_{i} T_{i}$. It follows from the QYBE that the $Y_{i}$ 's commute pairwise. Recall that we denote by $\tilde{Y}$ the product $\tilde{Y}=\prod_{i} Y_{i}$.

\section{Proposition 27.}

$$
\tilde{Y}=\left(\pi_{V} \otimes \cdots \otimes \pi_{V} \otimes \partial_{\triangleleft}\right)(\Delta^{(n)}\left(\nu^{-1}\right) \underbrace{(\nu \otimes \cdots \otimes \nu)}_{n+1}) .
$$

Proof. This expression for the iterated coproduct of $\nu$ in terms of the double-braidings is essentially its defining property. 
QUANTUM D-MODULES, ELLIPTIC BRAID GROUPS AND DOUBLE AFFINE HECKE ALGEBRAS 13
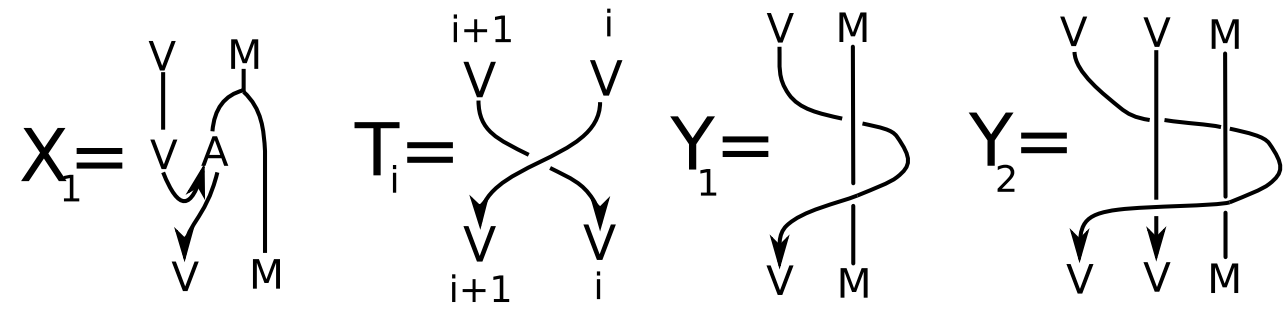

Figure 5. The operators $X_{1}, T_{i}, Y_{1}, Y_{2}$.

Recall that since $V$ is an $A$-comodule, we have the operators $L \in A \otimes E n d_{\mathbb{C}}(V)$ (see Prop 14). We can define $X_{1}=L_{01}$. Since $\Delta_{0}\left(L_{01}\right)=L_{02} L_{12}$, we have that $S_{0}\left(L_{01}\right)$ gives an inverse $X_{1}^{-1}$ (Here $S_{0}$ means that we apply the antipode in the $A$ component). We set $X_{i+1}=T_{i} X_{i} T_{i}$, for $i=1, \ldots, n-1$. The reflection equation implies that the $X_{i}$ commute pairwise. The invariant description for $X_{1}$ is depicted in figure 5, and it is perhaps more enlightening. We first comultiply $V$ as an $A$-module, then multiply the extra $A$-factor into $M$.

Lemma 28. $X_{i}, Y_{i}, T_{i}$ preserve the subspace of ad-invariants.

Proof. Note that $Y_{i}$ and $T_{i}$ are endomorphisms of the $U$-module $V^{\otimes n} \otimes M$. Thus, if $v \in\left(V^{\otimes n} \otimes M\right)^{i n v}$, we have that $x T_{i} v=T_{i} x v=\epsilon(x) T_{i} v$, and likewise for $Y_{i}$.

Only $X_{i}$ requires some computation. Since $X_{i+1}=T_{i} X_{i} T_{i}$, we only need to check for $X_{1}$. Since $D_{U}$ is a module-algebra under the adjoint action, it follows that for any $x \in U$, we have

$$
\begin{aligned}
(a d x) X_{1}\left(\sum_{i} v_{n}^{i} \otimes \cdots \otimes v_{1}^{i} \otimes m^{i}\right) & =(a d x) \mu_{21,0}\left(\sum_{i} v_{n}^{i} \otimes \cdots \otimes e_{k}^{i} \otimes e_{k}^{i *} \otimes v_{1}^{i} \otimes m^{i}\right) \\
& =\mu_{21,0}(a d x)\left(\sum_{i} v_{n}^{i} \otimes \cdots \otimes e_{k}^{i} \otimes e_{k}^{i *} \otimes v_{1}^{i} \otimes m^{i}\right) \\
& =\epsilon(x) \mu_{21,0}\left(\sum_{i} v_{n}^{i} \otimes \cdots \otimes e_{k}^{i} \otimes e_{k}^{i *} \otimes v_{1}^{i} \otimes m^{i}\right) \\
& =\epsilon(x) X_{1}\left(\sum_{i} v_{n}^{i} \otimes \cdots \otimes v_{1}^{i} \otimes m^{i}\right)
\end{aligned}
$$

We used that $U$ acts trivially on $e_{k} \otimes e_{k}^{*}$, as it is just the image of $1 \in \mathbb{C}$ under the coevaluation, and that the multiplication in $D_{U}$ is covariant for the adjoint action of $U$.

Proposition 29. $X_{1} Y_{2}=Y_{2} X_{1} T_{1}^{2}$.

Proof. We use remark 6 to express both sides as a braid diagram in figure 6 , at which point the equality is simply an identity of tangles.

This relation appears in a different form in [VV], where it is computed using the Fourier transform isomorphism: $\mathcal{F}: A \rightarrow U^{\prime}$. Recall that $U^{\prime}$ is the sub-algebra of $U$ which is locally finite under the adjoint representation. In our case, $\mathcal{F}$ applied to the zeroeth factor 


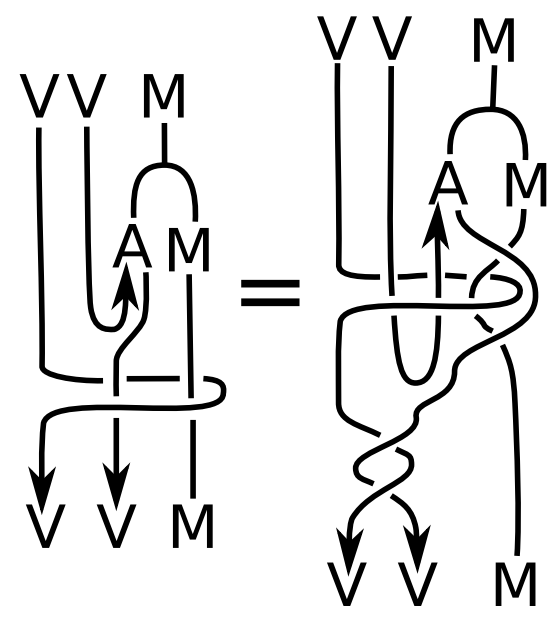

Figure 6. Proof of Proposition 29

maps the action of $X_{1}$ onto that of $Y_{1}$, and thus lines up with the Fourier transform of the torus. Expressing $X_{1}$ in terms of the co-product of $A$ allows us to give a diagrammatical proof instead.

Proposition 30. $\tilde{Y} X_{j}=X_{j} \tilde{Y}$

Proof. Actually, we'll show that $\tilde{Y}$ acts by $\left.(\nu \otimes \ldots \otimes \nu)\right|_{V \otimes n}$, so that it clearly commutes with $X_{j}$. We have by corollary 27 that

$$
\begin{aligned}
\tilde{Y}\left(\sum_{i} v_{n}^{i} \otimes \cdots \otimes v_{1}^{i} \otimes m^{i}\right) & =\Delta^{(n)}\left(\nu^{-1}\right)\left(\sum_{i} \nu v_{n}^{i} \otimes \cdots \otimes \nu v_{1}^{i} \otimes \partial_{\triangleleft}(\nu) m^{i}\right) \\
& =\sum_{i} \nu v_{n}^{i} \otimes \cdots \otimes \nu v_{1}^{i} \otimes \partial_{\triangleleft}(\nu) \partial_{\triangleright}\left(S^{-1}\left(\nu^{-1}\right)\right) m^{i}, \text { by ad invariance, } \\
& =\sum_{i} \nu v_{n}^{i} \otimes \cdots \otimes \nu v_{1}^{i} \otimes m^{i},
\end{aligned}
$$

by remark 9, and the fact that $S^{-1}(\nu)=\nu$. Notice that this is the only place in the argument where we use ad-invariance. Because $\nu$ is central, it is in $U^{\prime}$. Also it is easy to see that $\Delta^{(k)}\left(U^{\prime}\right) \subset U^{\otimes n} \otimes U^{\prime}$. Together, these justify shifting the ribbon element to the zeroeth component.

We have confirmed the necessary relations on the operators $X_{i}, Y_{i}, T_{j}$, which concludes the proof of Theorem 22 .

\section{A RELATiOn FOr $\tilde{X}$ AND $\tilde{Y}$}

As an immediate corollary of the proof of Proposition 30, we have the following Corollary 31. Suppose that $V$ is irreducible, and that $\left.\nu\right|_{V}=c_{V} i d$. Then $\tilde{Y}=c_{V}^{n} i d_{W}$. 
It turns out that $\tilde{X}:=\prod_{i} X_{i}$ acts by the same scalar. We prove this in two steps. Recall our standing assumption that $U$ has enough finite dimensional representations, in the sense of Definition 12,

Proposition 32. $\tilde{X}=(\tilde{T} \otimes 1 \otimes 1) \circ \mu_{A, M} \circ \Delta_{V^{\otimes n}}$, where $\tilde{T}: V^{\otimes n} \rightarrow V^{\otimes n}$ is the double braiding, $\tilde{T}=\prod_{i=1}^{n-1} \prod_{j \leq i} T_{j}$, depicted in figure 7 .

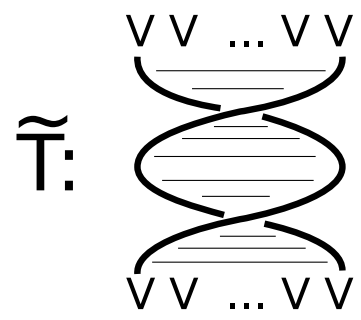

FigURE 7. $\tilde{T}$ braids all $n$ copies of $V$ twice around one another.

Proof. This is a direct computation, of which the reflection equation (14) is the case $n=2$.

Proposition 33. Suppose that $V$ is irreducible. Then $\tilde{X}=c_{V}^{n}$, where $c_{V}$ is the same as from Proposition 30.

Proof. We first consider the case $n=1$. Since we consider $U$-invariants, this is the same as $A$-coinvariants, and so we have that

$$
\begin{aligned}
\Delta_{V} & =\sigma_{M, A}^{-1} \circ\left(\mathrm{id} \otimes \mathrm{id} \otimes S^{-1}\right) \circ \Delta_{A}^{a d}, \text { and thus, } \\
\tilde{X} & =\mu_{A} \circ \sigma_{M, A}^{-1} \circ\left(\mathrm{id} \otimes \mathrm{id} \otimes S^{-1}\right) \circ \Delta_{A}^{a d},
\end{aligned}
$$

where $\Delta_{A}^{a d}$ is the co-adjoint action of $A$ on itself. In figure 8 , we compute that equation 2 is equal ad $(\nu)$ acting on $M$. For concreteness, we work in $M=A$, the basic representation of $D_{U}$, which we may do because this representation is faithful, by Theorem 17, and our assumption that $U$ has enough finite dimensional representations.

By invariance, $\operatorname{ad}(\nu)$ acting on $M$ is the same as $S(\nu)=\nu$ acting on $V$, as desired. When $n \geq 1$, we apply this proof to $V^{\otimes n}$ instead, to conclude that

$$
\begin{aligned}
\tilde{X} & =(\tilde{T} \otimes 1 \otimes 1) \circ \mu_{A, M} \circ \Delta_{V} \otimes n \\
& =\tilde{T} \circ \Delta^{(n-1)}(\nu) \otimes 1 \otimes 1, \text { by the } n=1 \text { case above. } \\
& =\left.(\nu \otimes \cdots \otimes \nu \otimes 1 \otimes 1)\right|_{(V \otimes n \otimes A)^{i n v}} \\
& =c_{V}^{n} \mathrm{id}
\end{aligned}
$$

Corollary 34. Let $B_{n}^{\text {ell,0 }}$ be the quotient group of $B_{n}^{\text {ell }}$ by the relations $\tilde{X}=\tilde{Y}=1$, and $\mathcal{H}_{n}^{0}(q, t)$ be the quotient of $\mathcal{H}_{n}(q, t)$ by the same relations. Setting $X_{i}^{\prime}=X_{i} c_{V}^{-1}, Y_{i}^{\prime}=Y_{i} c_{V}^{-1}$, 
we obtain an action of the group $B_{n}^{\text {ell, }, 0}$ on $W$, which descends to a representation of $\mathcal{H}_{n}^{0}(q, t)$ when $V$ is Hecke.

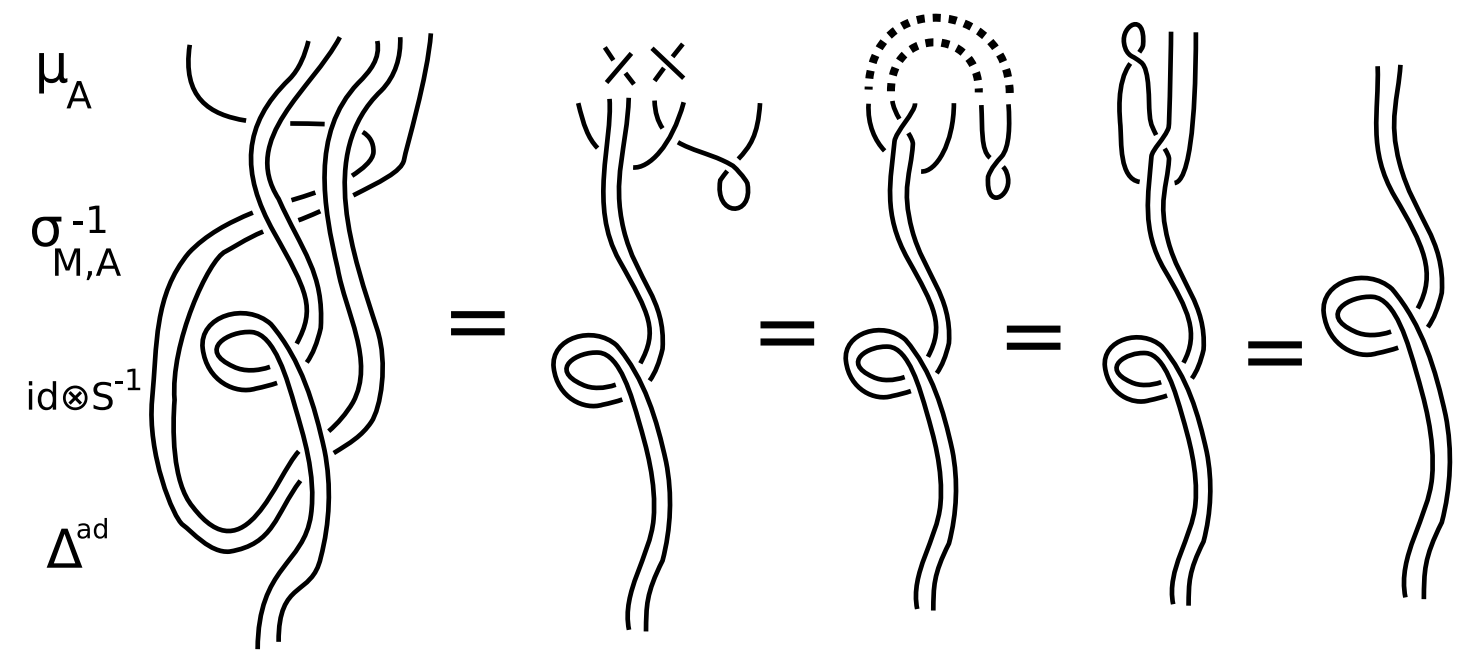

Figure 8. Computation of $\tilde{X}$. The second equality applies Proposition 10 to the braiding, as depicted above the diagram. The third equality applies Proposition 10 to the braiding composed with the coevaluation, as indicated by the dotted lines. The first and fourth equalities are already clear at the level of braiding diagrams.

\section{DAHA CASE AND ITS DEGENERATION}

In this section, we consider in more detail the case $U=U_{t}\left(s l_{N}\right)$. We recall the degeneration process from the DAHA to trigonometric Cherednik algebra, and we show that our construction degenerates to the construction of [CEE] (with trivial modifications) in the quasi-classical limit.

\subsection{A representation of $\mathcal{H}_{n}(q, t)$.}

Proposition 35. Let $V$ be the defining representation (of highest weight $(1,0, \ldots, 0)$ ) for $U_{t}\left(s l_{N}\right)$, and let $M$ be a $D_{U}$-module. Then the operators $X_{i}, Y_{i}$, and $T_{i}$ of the preceding section define a representation of $\mathcal{H}_{n}(q, t)$ with parameters $q, t=q^{n k}, k=N / n$ on the space $W=\left(V^{\otimes n} \otimes M\right)^{i n v}$, of invariants with respect to the adjoint action.

Proof. It is well known that the operators $\sigma_{V, V}$ satisfy the Hecke relation

$$
\left(\sigma_{V V}-t^{1-1 / N}\right)\left(\sigma_{V V}+t^{-1-1 / N}\right)
$$

which is the only additional relation on $\mathcal{H}_{n}(q, t)$ when $t=q^{n k}, k=N / n$. 
6.2. The trigonometric construction. In CEE, the authors constructed a representation of the trigonometric Cherednik algebra $\mathcal{H}_{n}^{\text {deg }}(k)$ on the space $\left(V^{\otimes n} \otimes M\right)^{i n v}$ of invariants with respect to the adjoint action, where $V$ is the vector representation, and $M$ is a D-module on $G$. The operators $s_{i j}$ were defined by the usual symmetric group action on $V^{\otimes n}$, while the $X_{i}, y_{j}$ were defined by:

$$
\begin{aligned}
X_{i} & =\sum_{r, s}\left(E_{s}^{r} \otimes A_{r}^{s}\right)_{i, 0} \\
y_{j} & =k\left(\sum_{p}\left(b_{p} \otimes L_{b_{p}}\right)_{j, 0}+\sum_{i<j} s_{i j}\right),
\end{aligned}
$$

where $A_{r}^{s}$ denotes multiplication by the function $A_{r}^{s}$ in the coordinate algebra, $\left\{b_{p}\right\}$ are a orthonormal basis with respect to the trace form on $s l_{N}$, and $L_{b_{p}}$ denotes the action by left translation along the vector field given by $b_{p}$.

6.3. Trigonometric degeneration of the DAHA. We recall the process of degeneration from the DAHA to the trigonometric Cherednik algebra. Let $\hbar$ denote a formal variable, let $k \in \mathbb{C}$, and let $q, t \in \mathbb{C}[[\hbar]]$ denote the power series

$$
q=e^{\hbar}, t=e^{n k \hbar} \text {. }
$$

Definition 36. $\widetilde{\mathcal{H}}_{n}$ is the $\mathbb{C}$-algebra freely generated by $X_{i}^{ \pm 1}, y_{i}, s_{j}$, for $i=1, \ldots, n$, and $j=1, \ldots, n-1$. Let $Y_{i}, T_{j} \in \widetilde{\mathcal{H}}_{n}[[\hbar]]$ denote the power series

$$
Y_{i}=e^{\hbar y_{i}}, \quad T_{j}=q^{-1} s_{j} e^{\hbar k s_{j}} .
$$

Let $\tilde{I}$ denote the closed ideal in $\widetilde{\mathcal{H}}_{n}[[\hbar]]$ generated by the DAHA relations from Definitions 1 and 2, and let $I$ denote the saturation of $\tilde{I}$ with respect to $\hbar$,

$$
I=\left\{x \in \widetilde{\mathcal{H}}_{n}[[\hbar]] \mid \hbar^{m} x \in \tilde{I} \text { for some } m \geq 0\right\} .
$$

Definition 37. $\widehat{\mathcal{H}}_{n}(q, t)$ is the quotient:

$$
\widehat{\mathcal{H}}_{n}(q, t)=\widetilde{\mathscr{H}}_{n}[[\hbar]] / I \text {. }
$$

Definition 38. $\mathcal{H}_{n}^{f}(q, t)$ is the complete $\mathbb{C}[[\hbar]]$-subalgebra of $\widehat{\mathcal{H}}_{n}(q, t)$ generated by $X_{i}, Y_{i}$, $i=1, \ldots, n$, and $T_{j}, j=1 \ldots, n-1$. It is a formal version of $\mathcal{H}_{n}(q, t)$ from Definition 2 ,

Proposition 39. Let $V$ be a representation of $\mathcal{H}_{n}^{f}(q, t)$ which is flat as a $\mathbb{C}[[\hbar]]$-module, and $\rho: \mathcal{H}_{n}^{f}(q, t) \rightarrow \operatorname{End}(V)$ the corresponding map. Then $\rho$ extends to a representation of $\widehat{\mathcal{H}}_{n}(q, t)$ if and only if $\rho\left(Y_{i}\right)=1 \bmod \hbar, \forall i$. In this case the extension is unique.

Proof. $(\Rightarrow)$ is obvious. To show $(\Leftarrow)$, we need to define the action of $y_{i}$ and $s_{i}$ on $V$. First we observe that since $s_{i}^{2}=1$, the identity $T_{i}=q^{-1} s_{i} e^{\hbar k s_{i}}$ can simply be solved for $s_{i}$ :

$$
s_{i}=\frac{q T_{i}-\sinh (\hbar k)}{\cosh (\hbar k)} \text {. }
$$


We can also solve the identity $Y_{i}=e^{\hbar y_{i}}$ for $y_{i}$ :

$$
y_{i}=\frac{1}{\hbar} \log \left(1-\left(1-Y_{i}\right)\right)
$$

where the RHS is a well-defined power series in $\hbar$ because we assumed $1-Y_{i}$ was divisible by $\hbar$. Uniqueness follows because we have explicitly solved for the $y_{i}$ 's and $s_{i}$ 's.

6.4. Trigonometric degeneration of the construction of section 4. In this section, we again consider $t, q$ as elements of $\mathbb{C}[[\hbar]]$, as in equation (3). We have the following well-known proposition

Proposition 40. Let $U_{1}=U_{t} / \hbar U_{t}$. Then $U_{1} \cong U\left(s l_{N}\right)$, the classical enveloping algebra. Furthermore, $U_{t}$ is a flat deformation of $U_{1}$.

Corollary 41. Let $D_{t}=D_{U_{t}}$ and $D_{1}=D_{t} / \hbar D_{t}$. Then $D_{1} \cong D_{U\left(s l_{N}\right)}$, and $D_{t}$ is a flat (in fact, trivial) deformation of $D_{1}$.

Proof. Indeed, we have that the Hochschild cohomology of $D_{G}$ is determined by the singular cohomology of $G: H H^{i}\left(D_{G}, D_{G}\right)=H^{i}(G, \mathbb{C})$. Thus, when $G$ is semi-simple, we have in particular that $H H^{2}\left(D_{G}, D_{G}\right)=H^{2}(G, \mathbb{C})=0$, so that $D_{G}$ admits no non-trivial formal deformations (see e.g, E] $)$.

Definition 42. Let $C$ denote the Casimir element $C=\sum_{p} b_{p} b_{p} \in U\left(s l_{N}\right)$, where $\left\{b_{p}\right\}$ form an orthonormal basis with respect to the trace form. Let $\Omega$ denote the canonical 2-tensor in $U\left(s l_{N}\right) \otimes U\left(s l_{N}\right)$,

$$
\Omega=\frac{\Delta(C)-C \otimes 1-1 \otimes C}{2}=\sum_{p} b_{p} \otimes b_{p} .
$$

Definition 43. Let $r$ denote the classical $r$-matrix for $U\left(s l_{N}\right)$, so $\Omega=r_{10}+r_{01}$

Proposition 44. We have the following relations between $r, R, R_{10} R_{01}$, and $\Omega$.

$$
\begin{aligned}
R & =t^{r}=1+k \hbar r \bmod \hbar^{2}, \\
R_{10} R_{01} & =t^{r_{10}} t^{r_{01}}=1 \otimes 1+k \hbar \Omega \bmod \hbar^{2} .
\end{aligned}
$$

Proposition 45. $\Omega$ acts as $\sigma_{V V}-1 / N$ on $V \otimes V$, the tensor square of the defining representation for $U\left(s l_{N}\right)$.

Proof. First we compute the canonical 2-tensor $\widetilde{\Omega}$ for $U\left(g l_{N}\right)$. Instead of an orthonormal basis $\left\{b_{p}\right\}$, we can choose the basis $E_{j}^{i}$ and dual basis $E_{i}^{j}$, and so

$$
\widetilde{\Omega}=\sum_{i, j} E_{j}^{i} \otimes E_{i}^{j}
$$

which is exactly the flip. Since $\Omega=\widetilde{\Omega}-1 / N\left(i d_{V} \otimes i d_{V}\right)$, the claim follows.

Proposition 46. The $\mathcal{H}_{n}(q, t)$-representation $W$ of Proposition 35 extends to a representation of $\widehat{\mathcal{H}}_{n}(q, t)$. 
QUANTUM D-MODULES, ELLIPTIC BRAID GROUPS AND DOUBLE AFFINE HECKE ALGEBRAS 19

Proof. $Y_{i}$ is expressed as a product of $R$-matrices, each of which is congruent to $1 \bmod \hbar$. Thus the condition of Proposition 39 is satisfied.

Proposition 47. Let $W_{1}=W / \hbar W=\left(V_{1}^{\otimes n} \otimes M_{1}\right)^{i n v}$. The operators $s_{i}$ act on $W_{1}$ as the flip $s_{i, i+1}$ of tensor factors. The operators $X_{i}$ act as

$$
X_{i}=\sum_{k, l}\left(E_{l}^{k}\right)_{i} \otimes A_{k}^{l} .
$$

Proof. Straightforward computation.

Proposition 48. The operators $y_{i}$ act as

$$
y_{i}=k\left(\Omega_{i, 0}+\sum_{j<i} s_{i j}-\frac{i-1}{N}\right)
$$

Proof. To see the claim for $y_{1}$, we note that

$$
Y_{1}=\left(R_{10} R_{01}\right)_{1,0}=1 \otimes 1+\hbar \Omega_{1,0} \quad \bmod \hbar^{2} .
$$

We proceed by induction:

$$
\begin{aligned}
Y_{i} & =T_{i} Y_{i-1} T_{i} \\
& =s_{i}\left(1+k \hbar r_{i, i-1}\right)\left(1+k \hbar\left(\Omega_{i-1,0}+\sum_{j<i-1} s_{i-1, j}-\frac{i-2}{N}\right)\right) s_{i}\left(1+k \hbar r_{i, i-1}\right) \bmod \hbar^{2} \\
& =1+k \hbar\left(\sum_{j<i-1} s_{i, j}+r_{i, i-1}+r_{i-1, i}+\Omega_{i, 0}-(i-2) / N\right) \bmod \hbar^{2} \\
& =1+k \hbar\left(\sum_{j<i-1} s_{i, j}+s_{i, i-1}-\frac{1}{N}+\Omega_{i, 0}-(i-2) / N\right) \bmod \hbar^{2} \\
& =1+k \hbar\left(\sum_{j<i} s_{i, j}+\Omega_{i, 0}-\frac{i-1}{N}\right) \bmod \hbar^{2}
\end{aligned}
$$

Comparing these with the operators of [CEE], we see that the quasi-classical limit of the present construction recovers the construction there, up to adding constants 4

\section{REFERENCES}

[AS] T. Arakawa, T. Suzuki, Duality between $\mathfrak{s l}_{n}(\mathbb{C})$ and the Degenerate Affine Hecke Algebra, Journal of Algebra 209, Academic Press, 1998.

[BK] B. Bakalov and A. Kirillov Jr, Lectures on Tensor Categories and Modular Functors, University Lecture Series, AMS 2001.

[Bir] J. Birman, On Braid Groups, Commun. Pure Applied Math. 22 (1969), 41-72.

\footnotetext{
${ }^{4}$ Also, our operators $L_{b}$ act by right instead of left translations, though this is just convention.
} 
[CEE] D. Calaque, B. Enriquez, and P. Etingof, Universal KZB equations I: the elliptic case, Preprint arXiv:math/0702670

[Ch] I.Cherednik, Double Affine Hecke Algebras, London Math. Soc. Lecture Notes Series 319.

[CBS] W. Crawley-Boevey and P. Shaw, Multiplicative preprojective algebras, middle convolution and the Deligne-Simpson problem, Adv. Math. 201 (2006), 180-208.

[E] P. Etingof, Cherednik and Hecke algebras of varieties with a finite group action, arXiv math.QA/0406499

[EFM] P. Etingof, R. Freund, and Xiaoguang Ma, A Lie theoretic constructino of representations of the degenerate affine and double affine Hecke algebras of type $B C_{n}$.

[EtGe] P. Etingof and Nathan Geer, Monodromy of the Trigonometric KZ equations, International Mathematics Research Notices (2007) Vol. 2007 : article ID rnm123, 15 pages, doi:10.1093/imrn/rnm123

[GG] W.L. Gan and V. Ginzburg, Almost-Commuting Variety, D-modules, and Cherednik Algebras, International Mathematics Research Papers (2006) Vol. 2006 : article ID 26439, 54 pages, doi:10.1155/IMRP/2006/26439

[Ka] C. Kassel, Quantum Groups, Springer Verlag, 1995.

[KiBa] B. Bakalov and A. Kirillov, Lectures on Tensor Categories and Modular Functors, AMS University Lecture Series, Vol 21, 2001.

[KlSch] A. Klimyk, K. Schmudgen, Quantum Groups and Their Representations, Springer, 1997.

[KrBa] E. Backelin and K. Kremnizer, Quantum Flag Varieties, equivariant quantum D-modules, and localization of quantum groups, Preprint arXiv:math/0401108

[Ly] V. Lyubashenko. Invariants of 3-manifolds and projective representations of mapping class groups via quantum groups at roots of unity, Comm. Math. Phys. 172 (1995) 467-516.

[LyMa] V. Lyubashenko and S. Majid, Braided Groups and Quantum Fourier Transform. J. Algebra 166, 506-528 (1994).

[Maj] S. Majid, Foundations of Quantum Group Theory, Cambridge University Press, 2000

[OS] A.A. Oblomkov, J.V. Stokman, Vector valued spherical functions and Mac- donald-Koornwinder polynomials, Compos. Math. 141 (2005), no. 5, pp. 13101350.

[VV] M. Varagnolo and E. Vasserot, DAHA at roots of unity, Preprint, arXiv:math/0603744

[VV2] M. Varagnolo and E. Vasserot, Schur duality in the toroidal setting, Comm. Math. Phys. Volume 182, Number 2 (1996), 469-483.

[Sc] G.P. Scott, Braid Groups and the group of homeomorphisms of a surface, Proc. Camb Phil. Soc. 68 (1970), 605-617. 